\title{
The effect of initial pressure on growth of FeNPs in amorphous carbon films
}

\author{
Fatemeh Mashayekhi ${ }^{1}$. Azizollah Shafiekhani ${ }^{2,3}$ - S. Ali Sebt ${ }^{1}$ - Elham Darabi ${ }^{1}$
}

Received: 20 February 2018 / Accepted: 6 March 2018 / Published online: 2 April 2018

(c) The Author(s) 2018

\begin{abstract}
Iron nanoparticles in amorphous hydrogenated carbon films (FeNPs@a-C:H) were prepared with RF-sputtering and RFPECVD methods by acetylene gas and $\mathrm{Fe}$ target. In this paper, deposition and sputtering process were carried out under influence of different initial pressure gas. The morphology and roughness of surface of samples were studied by AFM technique and also TEM images show the exact size of FeNPs and encapsulated FeNPs@a-C:H. The localized surface plasmon resonance peak (LSPR) of FeNPs was studied using UV-vis absorption spectrum. The results show that the intensity and position of LSPR peak are increased by increasing initial pressure. Also, direct energy gap of samples obtained by Tauc law is decreased with respect to increasing initial pressure.
\end{abstract}

Keywords FeNPs@a-C:H RF-sputtering and RFPECVD · AFM · TEM · LSPR

\section{Introduction}

In recent years, researchers showed more attention to nanomaterials because of wide variety of investigations and applications which can be considered for them [1-4]. Besides, nanoparticles or nanocrystals as a part of nanocomposite have been appeared in many studies to identify new aspects of their properties as new combination $[5,6]$.

Diamond like carbon (DLC) coating because of its special properties is one of the interesting subjects nowadays [7-10], while the first one was fabricated by Aisenberg and Chabot 50 years ago [11]. Some of the properties are low friction factor, high abrasion resistance, optical transparency, high hardness factor, heat stability, chemical neutral and biocompatibility [11,12]. High compressive stress of these carbon coatings makes a weak adhesion between layer and substrate; adding metal NPs to thin films of DLC (MeDLC) not only decreases the internal tension of carbon thin

Azizollah Shafiekhani

ashafie@ipm.ir

1 Department of Physics, Science and Research Branch, Islamic Azad University, P.O. Box 14665-678, Tehran, Iran

2 School of Physics, Institute for Research in Fundamental Sciences (IPM), P.O. Box 19395-5531, Tehran, Iran

3 Department of Physics, Alzahra University, Tehran 1993893973, Iran films but also increases some properties such as electric and magnetic properties [13].

Different methods like ion beam-assisted deposition (IBAD) and pulsed-laser deposition (PLD) have been applied to fabrication of Me-DLCs [13-15].

In the present work, for the first time, we simultaneously used radio frequency (RF) sputtering and RF plasma enhanced chemical vapor deposition (RF-PECVD) techniques to reach large amounts of iron nanoparticles in amorphous hydrogenated carbon films (FeNPs@a-C:H) [16-19]. Change in basic quantities such as initial pressure encouraged us to investigate this factor on properties of FeNPs@a$\mathrm{C}: \mathrm{H}$. Size distribution and roughness of FeNPs were studied using AFM and TEM images. Also, investigation on localized surface plasmon resonance (LSPR) shows an interesting comparison between two groups of studied samples in this work.

\section{Experimental details}

Iron nanoparticles were prepared in amorphous hydrogenated carbon films by co-deposition of RF-sputtering and RF-PECVD methods. In this method, the reactor is including two different electrodes which one is smaller than other. Iron plate is smaller which was applied as powered electrode and larger one was grounded via the chamber's body. 
All experiments were done at room temperature on quartz substrate and RF plasma system was activated by acetylene gas. The details of method can be seen in our pervious paper [10]. Two electrodes and RF power create plasma. The different areas of electrodes can produce a bias voltage $\left(V_{\mathrm{b}}\right)$ on the smaller electrode. This bias voltage is proportional to pressure $(P)$ and power supplied $(W)$ :

$V_{\mathrm{b}}=W / P^{1 / 2}$.

On the other hand, the $V_{\mathrm{b}}$ is directly related to the energy of the ions for sputtering according to the following relation:

$V_{\mathrm{b}}=k E_{i}$,

where $E_{i}$ and $k$ are ion energy and constant, respectively.

To study the effect of different pressures in prepared samples, they were divided into two groups based on deposition pressure. The particle size distribution and surface roughness of samples were all obtained by atomic force microscope (AFM) images in non-contact mode using a Nanoscope Multimode (Digital Instruments, Santa Barbara, CA), with a scan speed of $10-20 \mu \mathrm{m} / \mathrm{s}$. The exact size of the NPs and their shape in amorphous carbon film were observed by TEM images.

The LSPR peaks as optical properties of samples were detected using a UV-vis spectrometer in the $200-850 \mathrm{~nm}$ wavelength range.

\section{Results}

The properties of samples depend on the growth process of thin films. The pressure-time diagrams were plotted to study the growth process.

Figure 1 shows the treatment of pressure versus time deposition which results in a critical pressure about $20 \mu$ bar and leads to classify into two groups of samples. Group 1 and group 2 have pressures less and greater than $20 \mu \mathrm{bar}$, respectively. The details of prepared samples are given in Table 1 .

The variations of pressure inside the sputtering chamber were measured from initial moments of applying RF power.

The insert diagram of Fig. 1 represents the behavior of two groups in the early minutes of deposition.

Figure 1 shows the positive slope for samples 1 and 2 in group 1 at the first minutes. In this group, the free path length of hydrocarbon ions is high. Also, at low pressures, the bias voltage and the energy of ions are higher according to Eqs. (1) and (2). The sputtering process would be beginning with applied RF power, then the chamber's pressure increases to reach a maximum value of the mean free path length of the hydrocarbon ions and finally sputtering is stopped. At this moment, the hydrocarbon layers and

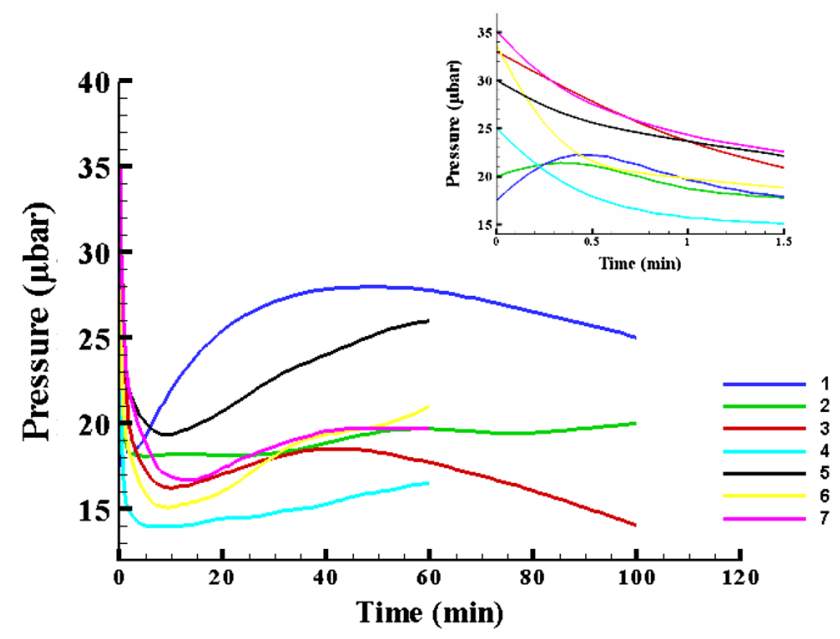

Fig. 1 Pressure-time diagram of groups 1 and 2. Insert diagram shows pressure-time diagram at 1.5 primary minutes of deposition

Table 1 Synthesis details of prepared samples

\begin{tabular}{lllll}
\hline Samples & $p(\mu \mathrm{bar})$ & $t(\min )$ & $W(\mathrm{w})$ & Group \\
\hline 1 & 17.5 & 100 & 280 & 1 \\
2 & 20.0 & 100 & 280 & 1 \\
3 & 33.0 & 100 & 280 & 2 \\
4 & 25.0 & $60+10$ & $240+80$ & 2 \\
5 & 30.0 & $60+10$ & $240+80$ & 2 \\
6 & 33.0 & $60+10$ & $240+80$ & 2 \\
7 & 35.0 & $60+10$ & $240+80$ & 2 \\
\hline
\end{tabular}

FeNPs were deposited simultaneously; therefore, the pressure of chamber is decreased.

According to Fig. 1, the pressure-time graph of group 2 was separated into two regions A and B that they had negative and positive slopes, respectively. In the first $10 \mathrm{~min}$, the pressure-time curve reduced with a large slope to a critical pressure between 14 and $20 \mu \mathrm{bar}$, the A region. The rate of deposition of carbon films is more than acetylene gas following into chamber, as the pressure of chamber decreased.

In this case, the mean free path length of ion hydrocarbons is increased. So, required energy for sputtering of target is provided. When sputtering from the Fe target started, the chamber pressure increased, but with a less slope than part A. The lower slope is due to the sputtering and deposition of FeNPs and hydrocarbons thin layers simultaneously. The deposited film on substrates has uniform thickness because of uniform plasma between two electrodes in the chamber.

To investigate the optical properties and prevent the rapid oxidation of FeNPs, some of the samples were deposited with carbon films. This deposition was carried out at a low power $(80 \mathrm{~W})$ during $10 \mathrm{~min}$, according to Table 1 . 
By AFM images, we investigate the effect of primary pressure for two examples of groups 1 and 2. Figure 2 shows the AFM images and topographies of samples 2 and 3 with 33 and $20 \mu$ bar initial pressures, respectively.

As is seen, the size of NPs is larger and the uniformity of their distribution is decreased with increasing initial pressure. Also, the surface roughness is increased (1.99 and $2.61 \mathrm{~nm}$ for samples 2 and 3, respectively).

The AFM images of each group were compared for more accurate studies on the effect of initial pressure on the NPs size and surface roughness. Figure 3 shows the AFM images of group 2 for samples 4, 5, 6 and 7 with initial pressure 25.0, 30.0, 33.0 and $35.0 \mu \mathrm{bar}$, respectively. As it can be seen, the size of NPs is larger and surface roughness is increased, with increasing initial pressure. The surface roughness of samples $4,5,6$ and 7 is $0.28,0.31,0.54$ and $0.75 \mathrm{~nm}$, respectively.

The deposition process shows an increase in the size of NPs by increasing initial pressure.

The mean free path of ions and consequently the energy of particles inside the chamber decreased when the pressure was increased. So the particles reach the substrate with less velocity and longer deposition time. In this case, FeNPs tend to accumulate on previous deposited ones. Thus, the size of formed NPs on substrate is larger and the surface roughness increased consequently.
Figure 4 shows the AFM images and size distribution of group 1, i.e., samples with pressure less than $20 \mu \mathrm{bar}$. In comparison, sample 1 with a lower initial pressure (17.5 bar) than sample 2 (20.0 bar) has uniform distribution size and smaller size of FeNPs.

In this method, carbon layers and NPs were deposited simultaneously, so the exact size of particles cannot obtain with the AFM images. On the other hand, this method has the advantage of stabilizing NPs by carbon layers, which prevents the agglomerated of the NPs.

We used TEM images to determine the exact size of NPs. Figure 5 shows TEM images with different scales and different points of sample 5. It is seen that FeNPs are spherical and well dispersed through carbon films.

Figure 5 h exhibits that average abundance of NPs size is $25 \mathrm{~nm}$ for Fig. 5a. The gray background in Fig. 5 is the carbon films. As a result, the deposition of carbon films and NPs simultaneously is an appropriate method for synthesis of FeNPs separately.

The optical properties of metal NPs are one of the unique properties which are different from the metal bulk completely. One of these optical properties is localized surface plasmon resonance (LSPR). LSPR depends on the type of material, size and shape of NPs and also environment dielectric function [20]. To obtain the FeNPs' LSPR, we used an UV-vis spectrometer in the range of $200-850 \mathrm{~nm}$.
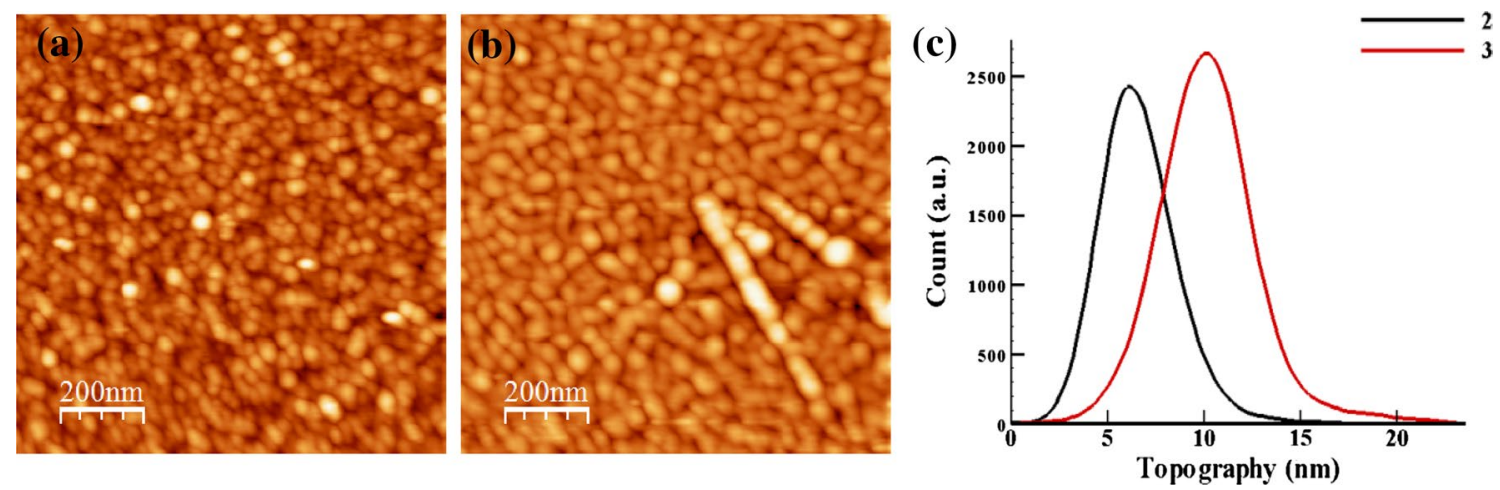

Fig. 2 a The AFM images of synthesized samples 2 (20.0 $\mu$ bar). b 3 (33.0 $\mu$ bar) and $\mathbf{c}$ topography of samples
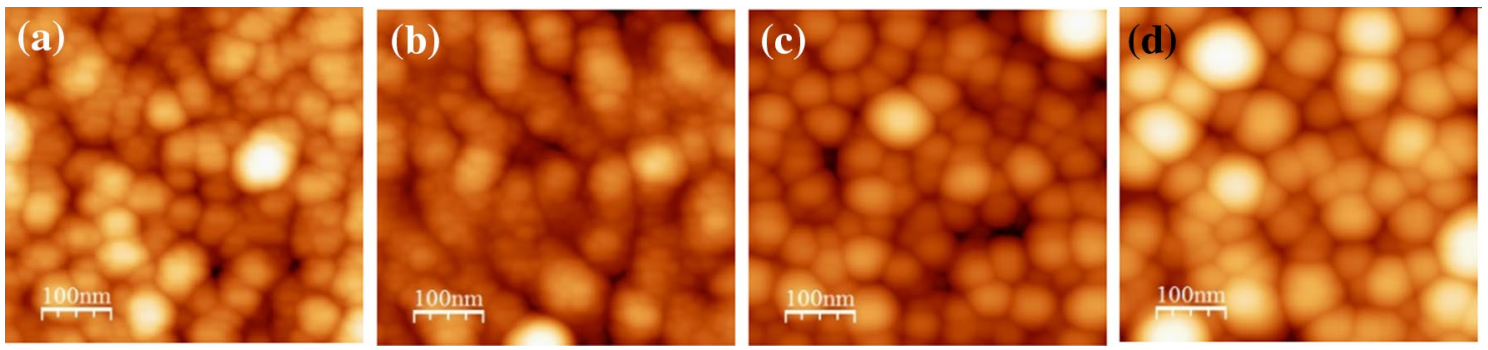

Fig. 3 The AFM images of samples a $4 \mathbf{b} 5 \mathbf{c} 6$ and $\mathbf{d} 7$ with initial pressure 25.0, 30.0, 33.0 and $35.0 \mu$ bar, respectively 
Fig. 4 a and b AFM images and c size distribution of samples 1 and 2
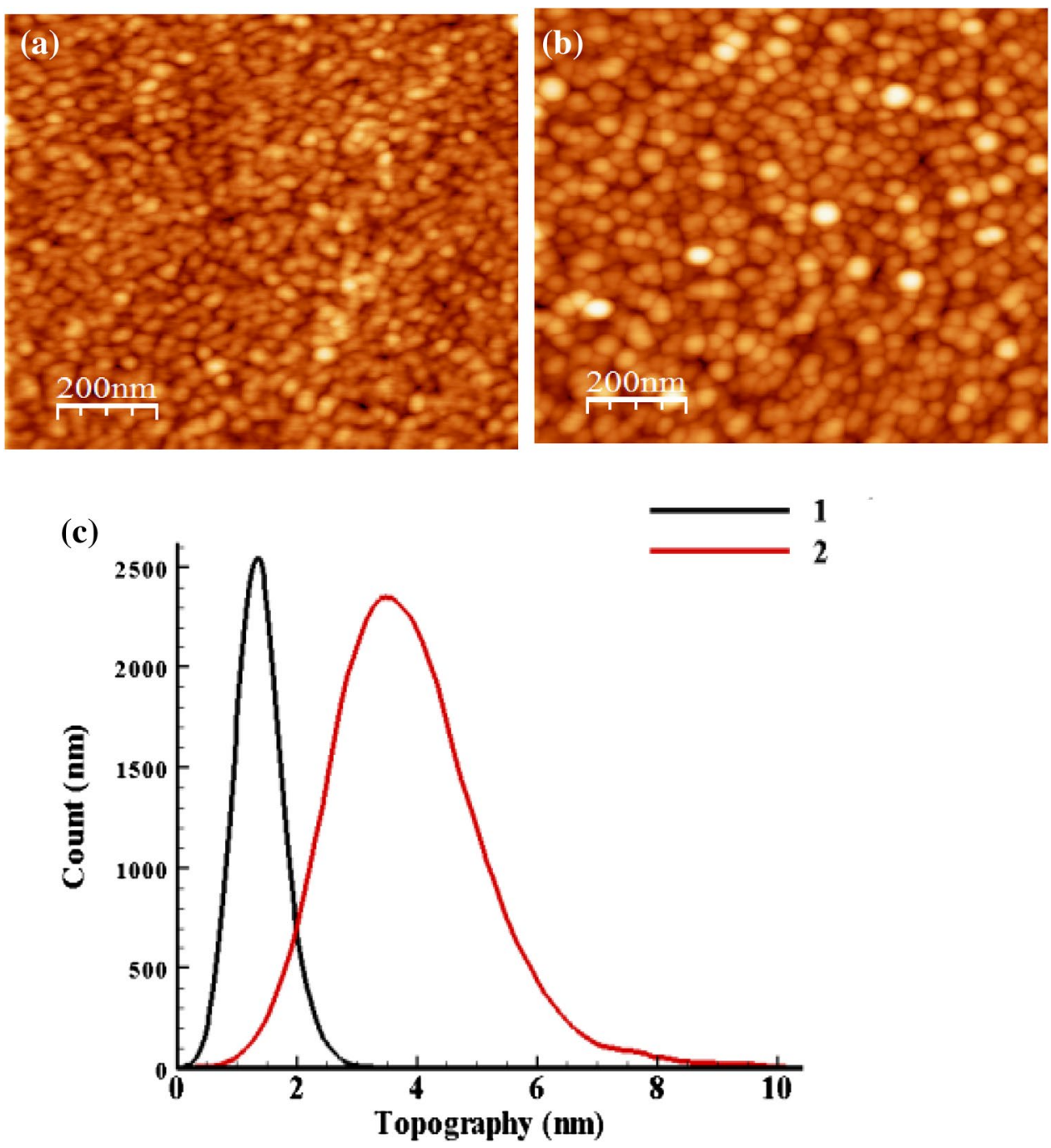

The spectrometer consists of two optical sources, one halogen-tungsten lamp and another deuterium lamp, which are in the visible and ultraviolet regions of electromagnetic spectrum, respectively.

The plasmonic peak of FeNPs is sharp compared to other elements (such as $\mathrm{Cu}, \mathrm{Au}, \mathrm{Ag}$ or Ni) [21]. Also, FeNPs have very high absorption of UV wavelengths; it means that these samples can be used as UV absorber windows.

The absorption spectra of samples of group 2 are shown in Fig. 6 and their LSPR peak data are given in Table 2. It is observed that the height, width and position of plasmonic peak increase with increasing initial pressure. On the other hand, the results of AFM images show that the size of particles is large and size distribution of them would be wider by increasing initial pressure. Therefore, plasmonic peak tends to larger wavelengths as red shift with increasing particle size. In fact, oscillation amplitude of electrons is greater when size of NPs becomes larger and their energy decreases. Therefore, position of LSPR peak occurs at larger wavelengths. Besides, by increasing the particle size distribution, plateau peak would be also wider since LSPR peak is a matrix of all LSPRs of NPs with different sizes. Hence, the sharp peak in plasmonic spectrum represents uniformity of NPs size.

The direct energy gap of samples obtained by UV-vis spectrum and Tauc law is calculated according to the following equation:

$(\alpha h v)^{2}=A\left(h v-E_{\mathrm{g}}\right)$,

where $h v$ is the photon energy, $E_{\mathrm{g}}$ is the optical energy gap and $A$ is the constant.

The results of these calculations are summarized in Table 2. The $E_{\mathrm{g}}$ decreased with increasing initial pressure. Considering AFM images, the size of NPs increased by increasing initial pressure; thus, energy gap reduced; this result is fully consistent with ones of Tauc law. 

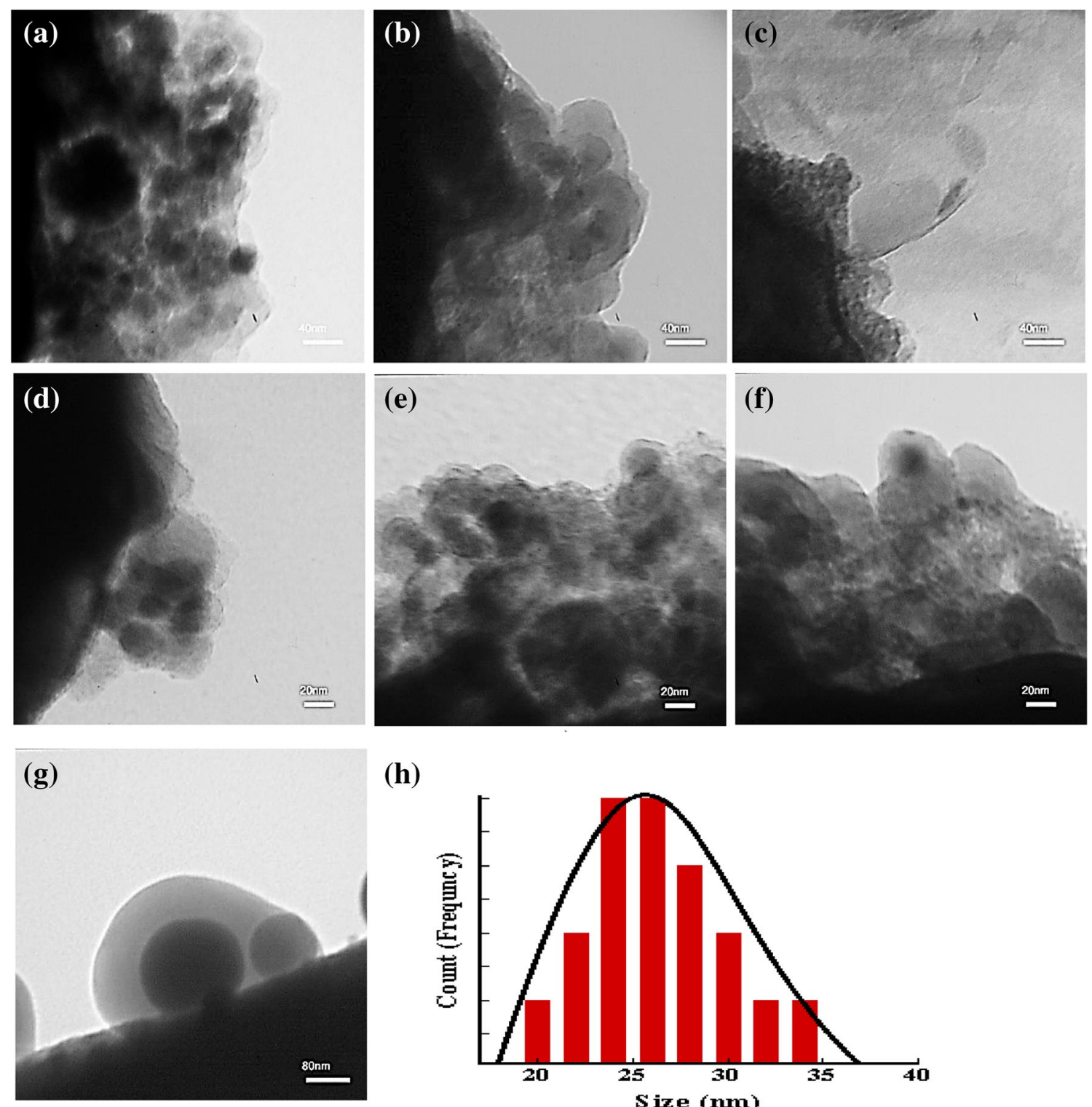

(h)

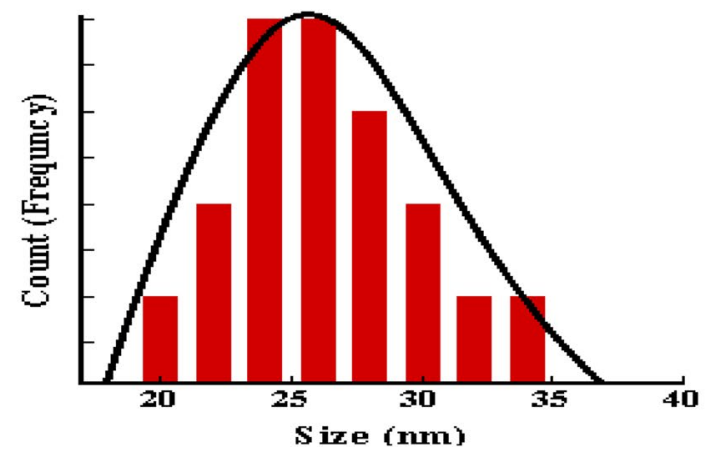

Fig. 5 a-g TEM images with different scales and different points of sample 5, h particle size distribution

\section{Conclusion}

FeNPs@a-C:H was fabricated using Fe target, acetylene gas, RF-sputtering and RFPECVD methods simultaneously. Pressure-time diagram introduces a critical pressure around $20 \mu$ bar which shows sputtering and NPs growth. Among lots of parameters like distance between electrodes, initial pressure of carrier gas, temperature and time of deposition, RF power, etc., that have an effect on the final properties of samples, the variations of initial pressure were also investigated. On the whole by increasing pressure, AFM images indicate that NPs size increases and distribution of size will be wider, also roughness increases. UV-vis spectroscopy in intensity and width of plasmonic peak increases and energy gap decreases.

Open Access This article is distributed under the terms of the Creative Commons Attribution 4.0 International License (http://creativeco mmons.org/licenses/by/4.0/), which permits unrestricted use, distribution, and reproduction in any medium, provided you give appropriate credit to the original author(s) and the source, provide a link to the Creative Commons license, and indicate if changes were made. 


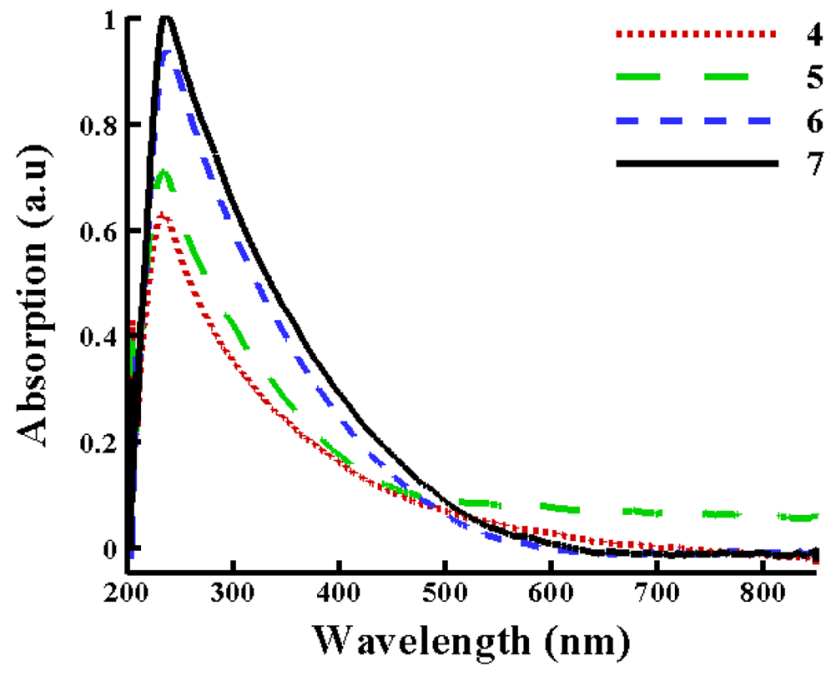

Fig. 6 The UV-vis absorption spectra of samples 4, 5, 6 and 7 with initial pressure $25,30,33.5$ and $35 \mu \mathrm{bar}$, respectively

Table 2 Height and position of LSPR and $E_{\mathrm{g}}$ of samples group 2

\begin{tabular}{lllll}
\hline Samples & pi $(\mu$ bar $)$ & Height (a.u) & $\lambda_{\text {LSPR }}(\mathrm{nm})$ & $E_{\mathrm{g}}(\mathrm{eV})$ \\
\hline 4 & 25.0 & 0.63 & 234 & 4.15 \\
5 & 30.0 & 0.72 & 235 & 3.94 \\
6 & 33.5 & 0.97 & 237 & 3.86 \\
7 & 35.0 & 1.00 & 238 & 3.84 \\
\hline
\end{tabular}

\section{References}

1. Țălu, Ş., Bramowicz, M., Kulesza, S., Ghaderi, A., Solaymani, S., Savaloni, H., Babaei, R.: Micromorphology analysis of specific 3 -D surface texture of silver chiral nanoflower sculptured structures. J. Ind. Eng. Chem. 43, 164-169 (2016)

2. Solaymani, S., Ghaderi, A., Dejam, L., Garczyk, Ż., Sapota, W., Stach, S., Dalouji, V., Luna, C., Elahi, S.M., Elahi, S.H.: Correlation between the multifractal structure, crystalline and photoluminescence properties of engineered CZO thin films. Int. J. Hydrogen Energy 42(20), 14205-14219 (2017)

3. Solaymani, S., Ghaderi, A., Beryani Nezafat, N.: Comment on: "Characterization of Microroughness Parameters in Titanium Nitride Thin Films Grown by DC Magnetron Sputtering" [J Fusion Energ DOI 10.1007/s10894-012-9510-z]. J Fusion Energy 31(6), 591 (2012)

4. Boochani, A., Nowrozi, B., Khodadadi, J., Solaymani, S., JalaliAsadabadi, S.: Novel graphene-like $\mathrm{Co}_{2} \mathrm{VAl}$ (111): case study on magnetoelectronic and optical properties by first-principles calculations. J. Phys. Chem. C 121(7), 3978-3986 (2017)

5. Ghodselahi, T., Solaymani, S., Pasha, M.A., Vesaghi, M.A.: Ni nanoparticle catalyzed growth of MWCNTs on Cu NPs@a-C:H substrate. Eur. Phys. J. D 66, 299 (2012)

6. Solaymani, S., Elahi, S.M., Nezafat, N.B., Zahrabi, H., Boochani, A., Naseri, M.: Characterization of microroughness parameters in $\mathrm{Cu}-\mathrm{C}$ nanocomposite prepared by co-deposition of RF-sputtering and RF-PECVD. Eur. Phys. J. Appl. Phys. 64, 11301 (2013)
7. Inoi, T., Baba, K., Flege, S., Hatada, R., Ensinger, W.: Preparation of iodine containing diamond-like carbon films by trifluoroiodomethane. Mater. Lett. 215, 68-70 (2018)

8. Pradeep, S., Surender, S., Prabakaran, K., Jayasakthi, M., Baskar, K.: Formation of graphitic and diamond-like carbon by low energy carbon ion implantation on c plane sapphire substrate. Thin Solid Films 649, 12-16 (2018)

9. Savchenko, D., Vorlíček, V., Prokhorov, A., Kalabukhova, E., Jelínek, M.: Raman and EPR spectroscopic studies of chromiumdoped diamond-like carbon films. Diam. Relat. Mater. 83, 30-37 (2018)

10. Ţălu, Ş., Bramowicz, M., Kulesza, S., Shafiekhani, A., Ghaderi, A., Mashayekhi, F., Solaymani, S.: Microstructure and tribological properties of FeNPs@aC:H films by micromorphology analysis and fractal geometry. Ind. Eng. Chem. Res. 54, 8212-8218 (2015)

11. Milewski, K., Kudlinski, J., Madej, M., Ozimina, D.: The interaction between diamond like carbon (DLC) coatings and ionic liquids under boundary lubrication conditions. Metalurgija 56, 55-58 (2017)

12. Ţălu, S., Stach, S., Ghodselahi, T., Ghaderi, A., Solaymani, S., Boochani, A.: Topographic characterization of $\mathrm{Cu}-\mathrm{Ni} \mathrm{NPs} @ \mathrm{aC}: \mathrm{H}$ films by AFM and multifractal analysis. J. Phys. Chem. B 119(17), 5662-5670 (2015)

13. Dimigen, H., Hübsch, H., Memming, R.: Tribological and electrical properties of metal-containing hydrogenated carbon films. Appl. Phys. Lett. 50, 1056-1058 (1987)

14. Hioki, T., Itoh, Y., Itoh, A., Hibi, S., Kawamoto, J.: Tribology of carbonaceous films formed by ion-beam-assisted deposition of organic material. Surf. Coat. Technol. 46, 233-243 (1991)

15. Tian, P., Zhang, X., Xue, Q.Z.: Enhanced room-temperature positive magnetoresistance of a-C:Fe film. Carbon 45, 1764-1768 (2007)

16. Dejam, L., Elahi, S.M., Nazari, H.H., Elahi, H., Solaymani, S., Ghaderi, A.: Structural and optical characterization of $\mathrm{ZnO}$ and AZO thin films: the influence of post-annealing. J. Mater. Sci. Mater. Electron. 27, 685-696 (2016)

17. Dalouji, V., Elahi, S.M., Ghaderi, A., Solaymani, S.: Influence of annealing temperature on berthelot-type hopping conduction mechanism in carbon-nickel composite films. Chin. Phys. Lett. 33, 057203 (2016)

18. Ţălu, Ş., Solaymani, S., Bramowicz, M., Kulesza, S., Ghaderi, A., Shahpouri, S., Elahi, S.M.: Effect of electric field direction and substrate roughness on three-dimensional self-assembly growth of copper oxide nanowires. J. Mater. Sci. Mater. Electron. 27, 9272-9277 (2016)

19. Ţălu, Ş., Bramowicz, M., Kulesza, S., Ghaderi, A., Dalouji, V., Solaymani, S., Khalaj, Z.: Microstructure and micromorphology of $\mathrm{Cu} / \mathrm{Co}$ nanoparticles: surface texture analysis. Electron. Mater. Lett. 12, 580-588 (2016)

20. Ţălu, Ş., Bramowicz, M., Kulesza, S., Solaymani, S., Shafikhani, A., Ghaderi, A.: Gold nanoparticles embedded in carbon film: micromorphology analysis. J. Ind. Eng. Chem. 35, 158-166 (2016)

21. Naderi, S., Ghaderi, A., Solaymani, S., Golzan, M.M.: Structural, optical and thermal properties of silver colloidal nanoparticles. Eur. Phys. J. Appl. Phys. 58(2), 377 (2012)

Publisher's Note Springer Nature remains neutral with regard to urisdictional claims in published maps and institutional affiliations. 